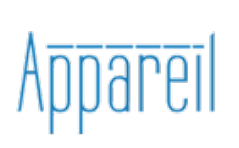

Appareil

4 | 2010

Biopolitique, éthique et subjectivation, questions de modernité

\title{
Ce que je dois à Foucault
}

Jean-Louis Déotte

\section{(2) OpenEdition}

Journals

Édition électronique

URL : https://journals.openedition.org/appareil/913

DOI : 10.4000/appareil.913

ISSN : 2101-0714

Éditeur

MSH Paris Nord

Référence électronique

Jean-Louis Déotte, «Ce que je dois à Foucault», Appareil [En ligne], 4 | 2010, mis en ligne le 27 janvier 2010, consulté le 12 octobre 2021. URL : http://journals.openedition.org/appareil/913 ; DOI : https:// doi.org/10.4000/appareil.913

Ce document a été généré automatiquement le 12 octobre 2021.

\section{(c) (i) () $\Theta$}

Appareil est mis à disposition selon les termes de la Licence Creative Commons Attribution - Pas d'Utilisation Commerciale - Pas de Modification 4.0 International. 


\title{
Ce que je dois à Foucault
}

\author{
Jean-Louis Déotte
}

1 J'ai commencé la lecture de Foucault avec Histoire de la folie à l'âge classique dès sa parution en 1972, dans des conditions idéales puisque j'étais enfermé dans la cour d'une caserne française afin d'y accomplir mon service militaire. Et les heures vides étant plus importantes que les exercices militaires, j'eus l'occasion de remplir deux cahiers de notes. Puis de retour à la vie civile, enseignant la philosophie dans une École normale, lieu de formation des instituteurs, je me jetais dans Surveiller et punir (1975). Or ces écoles pourraient passer pour des couvents catholiques du fait de leur architecture et parce que la discipline des corps et les valeurs morales imposées font de ces lieux dédiés à la laïcité (séparation de l'Église et de l'État, 1905) le verso de ce qu'elles sont censées combattre (la religion). Par ailleurs, j'habitais à quelques dizaines de kilomètres de la ferme où avait vécu la famille Rivière, lieu du crime perpétré par Pierre Rivière sur sa mère, sa sœur, son frère. Le film de René Allio, réalisé à partir des recherches de Foucault et de son équipe du Collège de France, sorti sur les écrans en 1976 eut un succès considérable dans cette région du bocage normand. C'est probablement au cours de ses déplacements à Caen, en particulier aux Archives départementales, que Foucault découvrit la prison de Beaulieu où fut interné Pierre Rivière. Or cette prison a la particularité architecturale d'être construite selon le principe du panopticon cher à Bentham, dispositif qui aura tant d'importance dans Surveiller et punir.

\section{1.}

2 On sait que la notion de dispositif, reprise dernièrement par Agamben (Qu'est-ce qu'un dispositif?, 2007) permet de synthétiser des séries discursives hétérogènes. En l'occurrence la série du savoir (les sciences humaines d'observation: psychiatrie, médecine, psychologie, etc.) et la série du pouvoir (agir sur les corps par l'enfermement afin d'influencer les âmes). M'intéressant essentiellement à l'esthétique, cette notion me permit de développer ce qui deviendra l'objet de ma thèse sur le musée comme origine de l'esthétique (1990, publiée en 1993). En effet, je trouvais chez Foucault la 
nécessité de l'institution comme lieu d'élaboration de nouveaux savoirs, voire comme lieu de surgissement d'une nouvelle réalité, ici les œuvres d'art. En effet, le musée, qui est une invention $d u \mathrm{XVIII}^{\mathrm{e}}$ européen, suspend des œuvres qui avaient auparavant une destination, cultuelle ou politique, pour, en les séparant en quelque sorte d'ellesmêmes, les produire dans la visibilité la plus générale, les rendant publiques. Il y a bien là production du nouveau qui est cohérent avec la définition de l'espace public par Kant dans sa Réponse à la Question: Qu'est-ce que les Lumières ? Cet espace public est rationnel en tant qu'espace de la communication et de la critique. Le statut des œuvres change en effet du tout au tout dans l'espace muséal : ce qui était de culte et donc communautaire devient public, ce qui était caché ou quasi-invisible dans un lieu de culte est livré à la lumière des débats du jugement esthétique; ce qui destinait des hommes à croire en l'au-delà devient l'objet d'une contemplation esthétique pour un sujet qui n'existait pas auparavant. On pouvait même ajouter que l'institution dans sa positivité avait précédé et rendu possible une nouvelle subjectivation ainsi que la philosophie de cette dernière (La Critique de la faculté de juger de Kant).

\section{2.}

Mais je ne tardais pas à me séparer de la notion de « dispositif » parce que s'il y a certes $\mathrm{du}$ « pouvoir » exercé dans les musées sur les œuvres, - et déjà cette violence qui fait qu'on arrache, souvent au cours de pillages militaires, les œuvres à leur lieux, les empêchant ainsi de destiner des communautés entières, ce que ne tarda pas à dénoncer un Quatremère de Quincy dans ses Considérations morales sur la destination des ouvrages de l'art (1815) -, mais parce qu'il y a aussi le pouvoir de légitimation exercé par le musée sur les œuvres qui est un mode de valorisation, il y a plus important. En effet, le musée est cette institution qui a la puissance de faire apparaître un nouvel objet: l'œuvre d'art; un nouveau sujet: le sujet esthétique; une nouvelle relation entre eux deux : la contemplation désintéressée. Dès lors sa puissance est plus significative du côté de l'apparaître : il configure différemment l'apparaître, donnant une autre définition à l'événement de ce qui paraît. Cette institution est un appareil: ce qui configure l'apparaître époque après époque. Cette définition de l'appareil nous contraint à donner une place essentielle à l'esthétique, et aux appareils qui ont successivement ébranlé et redéfini la sensibilité commune. La perspective est l'un des plus essentiels. Chez Foucault, cette dernière n'a pas cette centralité, même s'il donne une place considérable à certains de ses effets, en particulier à la place du spectateur devant un tableau. Or la perspective à point de fuite unique est au cœur de l'épistémè classique, parce qu'il n'y a pas de représentation sans perspective. C'est un point essentiel que Heidegger n'a pas vu dans sa critique de la Modernité (Qu'est-ce qu'une chose? L'époque des conceptions du monde, etc.).

\section{3.}

4 Le dispositif, par exemple le panopticon, va introduire la série du pouvoir dans un cadre qui ne concernait que les savoirs de représentation, en particulier la linguistique ( $L a$ Grammaire générale), le classement des espèces, l'économie de la monnaie. L'épistémè est un ensemble de normes soumettant la culture à un ensemble de contraintes qui, dans le cas présent, a supplanté l'épistémè de la Renaissance. L'analyse foucaldienne donne une 
place essentielle à la définition du signe. Ce signe est entendu comme représentation, c'est-à-dire comme tableau et carte, il est donc quasiment sans consistance, sans épaisseur, dédié à la dénotation. L'analyse de l'épistémè classique, développée dans Les Mots et les Choses, est jalonnée idéalement par Foucault par deux œuvres majeures : Les Ménines de Vélasquez, le Bar des Folies Bergères de Manet (l'étude sur Manet donnera lieu à une série de conférences, dont celle de Tunis en 1971 et fut publiée en 2004 par Maryvonne Saison). La seconde œuvre est l'inverse de la première, écrit-il. Ce sont deux œuvres qui ont affaire avec l'espace de représentation, ce sont deux scènes, mais alors que Les Ménines exhiberaient toutes les caractéristiques de la représentation, le tableau de Manet introduirait une complication (deux espaces de projection dans un seul tableau) annonçant une rupture interne.

Las Meninas, Diego Velasquez, 1656-1657

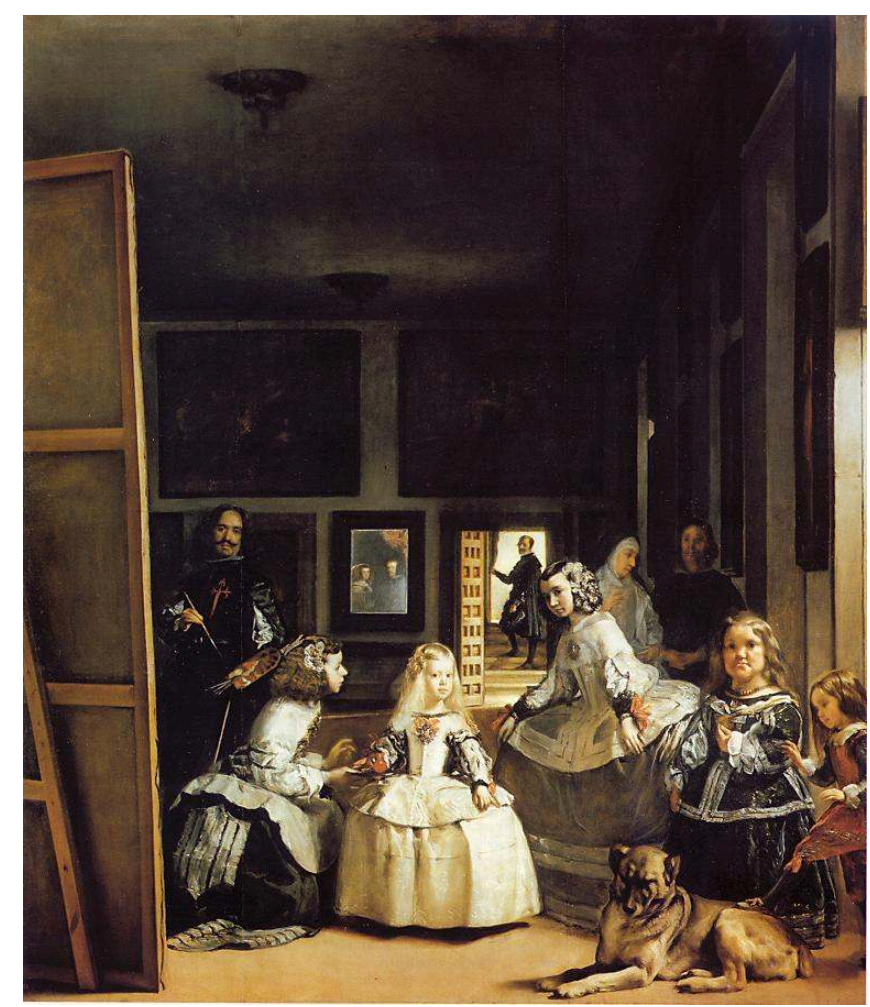

On peut être surpris que Foucault, s'attaquant à deux œuvres fortement structurées par la perspective, n'en donne qu'une lecture académique, comme on pourrait le faire pour n'importe quel tableau de n'importe quelle époque. J'ai parlé de la place du spectateur, qui est aussi celle du peintre, le point de vue idéal des traités de perspective. J'ajouterai à cela l'importance des surfaces réfléchies dans le tableau, métaphore du tableau comme miroir et fenêtre, de la place de la lumière, externe pour Les Ménines, frontale pour Manet, des lignes horizontales et verticales qui sont comme des éléments de cadre dans le cadre, je finirai, parce que c'est la clef de son dispositif herméneutique pour Les Ménines, par les jeux de regard des suivantes, de l'Infante, qui convergent vers l'avant et donc vers l'invisible et qui donnent toute son importance à la place du spectateur qui est aussi celle du roi en l'occurrence. J'ai parlé de lecture, parce que tout se passe comme si le tableau était un énoncé, ou une série d'énoncés. Il y va du spectateur, c'està-dire de l'homme comme sujet-objet des sciences humaines. De cet homme qui devrait être là à sa place, devant les Ménines, regardant une représentation, une scène de 
peinture où le peintre est bien assigné avec ses outils qui sont aussi ses marques de distinction (c'est un autoportrait) et au fond, dans un miroir, l'image spéculaire du couple royal, les commanditaires. Mais l'homme n'est pas à sa place. Dès lors, on peut penser que ce tableau qui est pourtant logé au cœur de l'épistémè classique, annonce sa fin, ce qu'une autre toile, le Bar des Folies Bergères de Manet viendra confirmer. Il s'agit donc d'un tableau précurseur d'une autre épistémè, cette autre épistémè où les tableaux ne seront plus de représentation, mais tels qu'en eux-mêmes, n'étant rien d'autre que ce qu'ils donnent eux-mêmes à voir, leur matérialité, selon la doctrine moderniste de Greenberg. L'invisibilité de l'homme des sciences humaines en train de se déployer annonce sa disparition, ce par quoi s'achève Les Mots et les choses.

Bataille quand il a écrit lui aussi sur Manet a mis l'accent sur le sacrifice du sujet. C'est beaucoup plus précis, parce qu'il n'identifie pas le sujet à la place du spectateur, ni au sujet de la narration, mais à ce qui rend possible une toile en perspective, à savoir le point de fuite des lignes, qu'on nomme dans les traités classiques (Desargues) : point $d u$ sujet. Voilà la grande affaire : c'est que le sujet n'est pas là où on croit qu'il est en général, à la place du spectateur, non, il est là-bas, au fond, là où les droites convergent dans la construction. Le sujet est un point idéal (point du sujet). Moi (le spectateur), je ne suis rien avant de regarder un tableau en perspective : la subjectivation n'a lieu que lorsque moi, le spectateur quelconque, je suis capté par cet œil de cyclope qui m'attend là-bas et qui, une fois qu'il m'a pris, ne me lâche plus, au point de me croire moi, finalement, un sujet ! La subjectivation est de fait un absorbement fort inquiétant, c'est une puissance d'emprise, où l'on ne dira pas que le moi s'aliène, car il n'y a encore rien de tel, mais c'est le pouvoir d'un appareil qui depuis qu'il s'impose aux apparaissants les leste de subjectivité.

7 Si Foucault, au lieu de s'attacher aux lignes des regards, avait tracé les lignes de fuite des Ménines, il aurait saisi qu'elles convergent vers cet étrange personnage au fond à droite, surélevé, qui soulève une lourde tenture, comme s'il dévoilait un secret: le secret du sujet. Il est peut-être le vrai souverain : cette bouche souveraine qui attend l'altérité pour la dévorer. 
Les lignes de fuite des Ménines

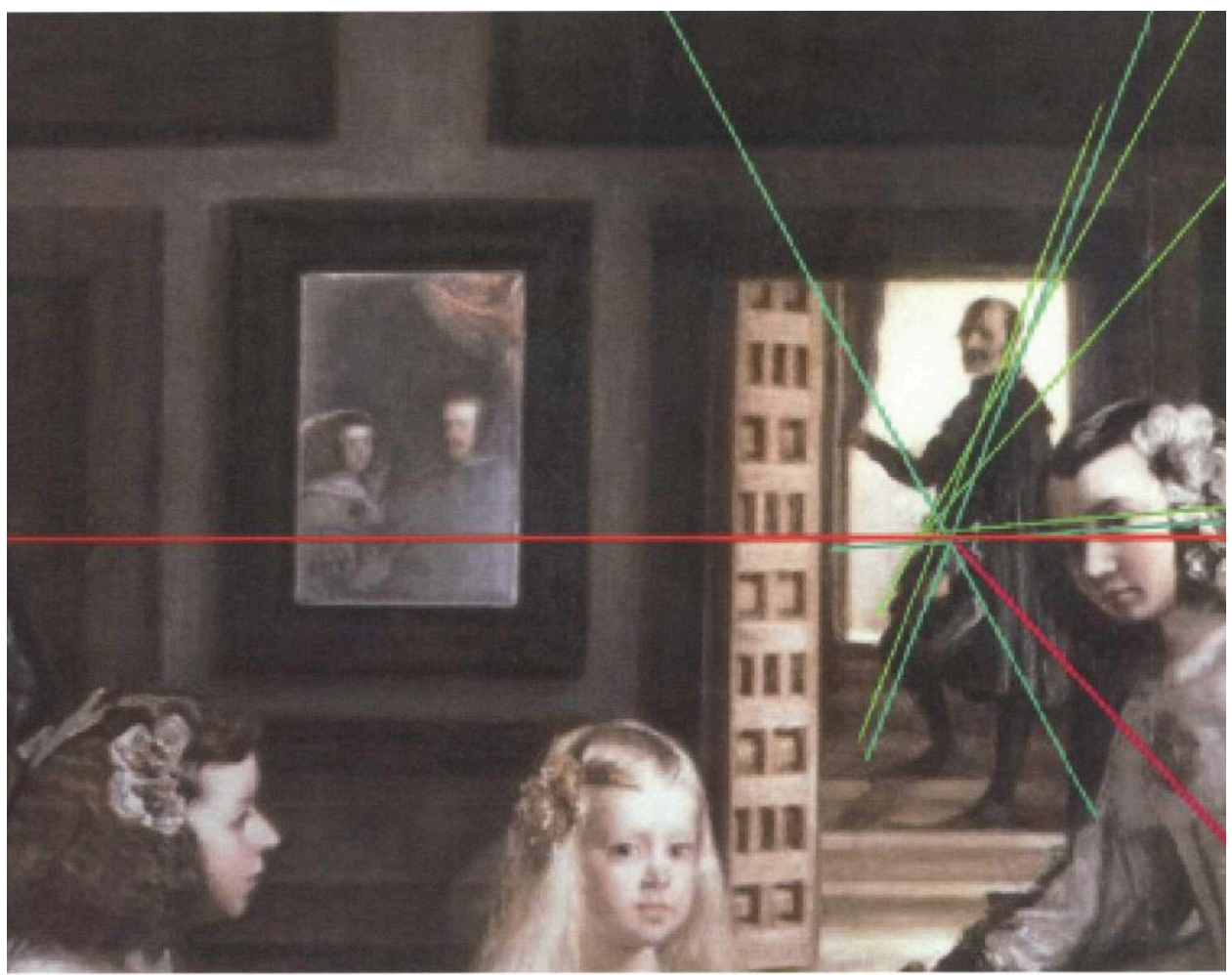

\section{4.}

8 Je suis aussi très sensible à l'interprétation que donne Foucault du texte de Kant: Qu'est-ce que les Lumières? Parce qu'il met l'accent sur la modernité comme attitude quant à l'époque où l'on vit et peut-être philosophique On sait que Baudelaire est pour lui, de ce point de vue, le premier des modernes. Être moderne consisterait pour un foucaldien à faire le point sur les dispositifs les plus actifs aujourd'hui, parce que l'action doit connaître les limites de son époque. Cette notion de limite est au cœur de la définition du transcendantal chez Kant: l'étude des conditions de possibilité du savoir et par voie de conséquence de ce qui lui est interdit. L'archéologie foucaldienne est une manière d'étude transcendantale ne se bornant pas aux limites du savoir, mais à celles de l'action. C'est dire que ces limites (configurations de la folie, de la maladie, des genres sexuels) sont factuelles, historiques et culturelles et doivent être dépassées.

9 Si le livre Les mots et les choses a été un énorme succès de librairie, a connu un effet de mode, il n'a peut-être pas été prolongé comme d'autres textes majeurs de Foucault, comme Surveiller et punir par exemple. Je ferai néanmoins l'hypothèse qu'on retrouverait une descendance inattendue chez le Lyotard de 1985 exposant, par le médium de l'exposition artistique, une idée spéculative, celle des Immatériaux. On peut faire l'hypothèse que dans cette exposition qui s'était déployée au Centre G. Pompidou, Lyotard a voulu rendre compte de l'épistémè de la postmodernité, en mettant l'accent sur la numérisation, les débuts d'Internet, la fabrique des corps artificiels, la fragmentation des savoirs et finalement l'indestination de l'humanité dite " postmoderne ». 


\section{BIBLIOGRAPHIE}

Agamben Giorgio, Qu'est-ce qu'un dispositif?, Paris, Payot \& Rivages, 2007.

Foucault Michel, Histoire de la folie à l'âge classique, Paris, Gallimard, 1972.

Foucault Michel, Surveiller et punir, Paris, Gallimard, 1975.

Foucault Michel, Les mots et les choses : une archéologie des sciences humaines, Paris, Gallimard, 1989.

Quatremère de Quincy Antoine, Considérations morales sur la destination des ouvrages de l'art, Paris, Fayard, 1989.

\section{RÉSUMÉS}

Foucault analyse l'épistémè classique (la représentation) à partir de deux peintures : Les Ménines de Vélazquez (Les Mots et les Choses) et Le Bar des Folies Bergères de Manet (Conférence de Tunis). La place du spectateur semble être la grande question, comme si la question du sujet était la même question. Mais, il y a une confusion, toute métaphysique : le sujet n'est pas le spectateur (point de vue) mais le point de fuite qu'en anglais on traduit judicieusement par vanishing point. Lequel se trouve en face du point de vue et n'est rien d'autre que le point de fuite. Pour approcher la question de la subjectivation, nous devons décrire l'appareil perspectif.

\section{AUTEUR}

\section{JEAN-LOUIS DÉOTTE}

Professeur des universités (philosophie esthétique), département de philosophie Paris 8 